\title{
Fichtes Wissenschaftslehre im Aufbrechen der Paradigmen mechanizistischen Denkens
}

\author{
Jürgen Stahl (Leipzig)
}

Ein in seiner Wertigkeit kaum zu überschätzendes Paradigma des sich auf die Erkenntnisse der Naturwissenschaften stützenden Weltbildes der europäischen Aufklärung lautete: "Natura non facit saltus". In der wissenschaftsgeschichtlichen Bewertung erfuhr es eine zuweilen unangemessene, weil überfordernde pejorative Einschätzung. Kriterium dafür war, daß diese Auffassung es nicht erlaubte, die Herausbildung der qualitativen Vielfalt des Seins in seiner Entwicklung theoretisch zu erfassen. Nur konnte ein solches Anliegen auf dem gegebenen Stand der Wissenschaftsentwicklung im 18. Jahrhundert als theoretisches Problem kaum hervortreten. Das in der These "Die Natur tut keine Sprünge" ausgesprochene Diktum ging natürlich nicht auf die Erklärung von Entwicklungszusammenhängen; es richtete sich auf die Abweisung von "Wunderglauben", der die Welt in ihren vielfältigen Erscheinungsformen als Werk Gottes erfaßte. Demgegenüber suchte die sich auf die Naturwissenschaften stützende Aufklärungsphilosophie in der Zurückdrängung deistischer Argumentationen alle Phänomene von einem "natürlichen", innerweltlichen Standpunkt aus als System gemäß dem Kontinuitätsprinzip zu erklären. Die universelle wechselseitige Determination sahen beispielsweise weder Leibniz, noch Wolff durch "plötzliche Begebenheiten" als dem Umschlagpunkt für qualitative Veränderungen durchbrochen. ${ }^{1}$ Eine solche, auf die Erfassung der Welt in ihrer Ganzheitlichkeit gehende Sicht, wie sie sich in der europäischen Aufklärung in philosophisch unterschiedlich begründeter Weise von Spinoza bis zu den französischen Enzyklopädisten oder den genannten deutschen Denkern darstellt, war aber in wissenschaftsgeschichtlicher Hinsicht nicht schon das Grab jeglichen auf die Genese der Erscheinungen zielenden Denkens. Insofern der naturwissenschaftlich begründete Nachweis der Interdependenz aller Erscheinungen zunehmend

\footnotetext{
${ }^{1}$ Vgl.: Ch. Wolff: Vernünfftige Gedancken von GOTT, der Welt und der Seele des Menschen. Leipzig 1719, §§ 686, 689 ganz im Sinne von Leibniz' "Gesetz der Stetigkeit" (vgl.: G.W. Leibniz: Neue Abhandlungen über den menschlichen Verstand. Hrsg. v. E. Cassirer. Leipzig 1915. S. 342), dessen Komplement "scheinbare Sprünge" als Erfordernis der Schönheit der Natur sind (ebenda, S. 573).
} 
auch das Problem ihres Auseinanderhervorgehens in neuer, einer den Mystizismus überwindenden Denkweise hervortreten ließ, gehört diese zu den Voraussetzungen für die Entfaltung darauf aufbauender Betrachtungen, die das Werden in den Blick nahmen. ${ }^{2}$

Der Leistungsfähigkeit zeitgenössischer wissenschaftlicher Theorien entsprechend wurde insbesondere auch in der deutschen Aufklärung versucht, die methodischen Prinzipien der Mathematik bzw. der klassischen Mechanik zu Allgemeinen zu erheben. Im Gegensatz zu dem aus der aristotelischen Denktradition herrührenden Methodenverständnis, wonach sich mathematische Erkenntnis auf den Bereich des Nichtstofflichen beziehe, andere Gegenstände folglich unter-schiedliche Methoden erfordern würden, ${ }^{3}$ ging das mit der Begründung des Theorienaufbaus gemäß dem "mos geometricus" verbundene Anliegen eben darauf aus, methodische Strenge und Verbindlichkeit für alle Wissenschaftsdisziplinen zu sichern. Dabei war dieses Methodenverständnis keineswegs auf das Auffinden von Begründungszusammenhängen restringiert. Als ars inveniendi verstanden ging es um die Gewinnung von Einsichten in die Art und Weise des Zusammenhanges, um auf unbekannte Größen schließen zu können.

Vorteil wie offensichtliche Grenze der enzyklopädischen Wissenssysteme der europäischen Aufklärung war, daß Mensch und Gesellschaft in die universell begriffene Naturgesetzlichkeit weitgehend eingegliedert waren. Dieses methodische Grundverständnis lag auch dem durch Lambert in der deutschen Aufklärung in herausragender Weise unternommenen Versuch des Aufbaus einer "mathesis universalis" zugrunde. Darin manifestierte sich nicht zuletzt das Anliegen, die Vielfalt des Seins nicht nur in ihrer quantitativen Dimension zu fassen, hatte doch bereits Leibniz die Idee einer "ars characteristica combinatoria" mit der einer universellen Zeichensprache verbunden, um die qualitative Spezifik der verschiedenen Seinsformen fassen und kalkülmäßig ableiten zu können. ${ }^{4}$ Lambert erneuerte diesen Anspruch, indem er die qualitative Seite der Begriffsbestimmungen mittels Zeichen für die gesamte Erkenntnis, sowohl ihrer Form als auch ihrem Inhalt nach, zu leis-

\footnotetext{
${ }^{2}$ Insofern gab Platner mit seiner Forderung nach einer "pragmatischen Geschichte des menschlichen Geistes" im Vorfeld der Ausbildung der Transzendentalphilosophie (vgl.: E. Platner: Philosophische Aphorismen nebst einigen Einleitungen zur philosophischen Geschichte. [Leipzig 1793] In: Akad.-Aus. Bd. II/4. S.12, 184) einem Problembewußtsein Ausdruck, das eine Stufe hinreichender Inventarisierung des Wissens als Voraussetzung erforderte.

${ }^{3}$ Vgl.: H.-W. Arndt: Methodo scientifica pertractatum. Mos geometricus und Kalkülbegriff in der philosophischen Theorienbildung des 17. und 18. Jahrhunderts. Berlin/New York 1971. S.3

4 Vgl.: Ebenda. S. 4
} 
ten bestrebt war. ${ }^{5}$ Ein nicht zu unterschätzendes Ergebnis der übergreifenden Methodenbegründung im Kontext der Bemühungen um eine "mathesis universalis" war die sich differenzierende Kenntnis der grundlegenden Formen logischen Denkens über die aristotelische Syllogistik hinaus, wodurch die Rolle des Subjekts im Erkenntnisprozeß stärker in das Blickfeld rückte.

Die Wissenschaftsgeschichte erwies die Undurchführbarkeit des in dieser Weise gestellten Anspruches. Der Problematik einer Mensch und Gesellschaft der Naturkausalität unterwerfenden Auffassung war man sich durchaus bewußt und suchte sie beispielsweise im Wolffschen System dadurch zu lösen, daß die in der Natur, dem organischen Leben und in der sozialen Sphäre erkennbare Zweckmäßigkeit durch ein über die Kausalität der Natur hinausgehendes "Mehr" erklärt wird: die in ihr liegende Fähigkeit zur Zwecktätigkeit. ${ }^{6}$ Daß diese Situation als unbefriedigend angesehen wurde, beweisen die durch die Berliner Akademie zwischen 1761 und 1807 mehrfach gestellten Aufgaben zum MethodenProblem.7 Zunehmend wurde deutlicher, daß weder die Philosophie noch andere aufkeimende Sozialwissenschaften und auch die Naturforschung, wo sie es mit Leben zu tun hat, nicht als bloßer Formalismus nach dem Modell der Mathematik oder Physik aufbaubar waren. Ebenso zeigten sich die Ansätze zu genetischem Denken nur bedingt in eine vom Ordnungswissen dominierte Naturforschung integrierbar. Doch weder die einzelwissenschaftlichen Ergebnisse, noch das den anerkannten Paradigmen der klassischen Mechanik bzw. Mathematik folgende Methodenverständnis vermochten das statische Weltbild auf diesem Stand der Wissenschaftsentwicklung ernsthaft ins Wanken zu bringen. ${ }^{8}$ Dazu bedurfte es einer methodologischen Grundlegung, die über die Interpretation einer bestimmten Vorgehensweise in der Wissenschaft hinausging.

\footnotetext{
${ }^{5}$ Vgl.: J.H. Lambert: Anlage zur Architectonic, oder Theorie des Einfachen und des Ersten in der philosophischen und mathematischen Erkenntniß. In: J.H. Lambert: Philosophische Schriften. Hrsg. v. H.-W. Arndt. 3.u.4.Bd. Hildesheim 1965. Vorrede. S. XXII

${ }^{6}$ Dazu näher: H. Poser: Die Einheit von Teleologie und Erfahrung bei Leibniz und Wolff. In: Formen teleologischen Denkens. H. Poser (Hrsg.): TUB-Dokumentation Kongresse und Tagungen. Berlin 1981. H.11. S. 110f.

7 Vgl.: H.-J. Engfer: Philosophie als Analysis. Studien zur Entwicklung philosophischer Analysiskonzeptionen unter dem Einfluß mathematischer Methodenmodelle im 17. und frühen 18. Jahrhundert. Stuttgart - Bad Cannstatt 1982. S. 26ff.; vgl. dazu: C. Buschmann: Philosophische Preisfragen und Preisschriften der Berliner Akademie 1747-1768. In: Deutsche Zeitschrift für Philosophie. 35.Jg. (1987) H.9. S. 779ff.

8 Dieser Situation ähnlich hatten sich in der Naturforschung des 18. Jahrhunderts bei der Bewältigung der organismischen Mannigfaltigkeit zwei kontroverse Linien herausgebildet: Die Konzeption der Gradation und Kontinuität (Leibniz/Enzyklopädisten) und die der Diskontinuität (Linné). Beiden war die Vorstellung der Konstanz und Statik der beste-
} 
Kant reagierte auf die Herausforderung dieser historischen Konstellation, indem er das Denken überhaupt, das Subjekt selbst in den Mittelpunkt transzendentalen Philosophierens stellte. Jedoch korrigierte schon Fichte die unbefriedigende Kantsche Lösung einer Alternative zwischen Determinismus und Indeterminismus, wonach der Mensch als Bürger zweier Welten einerseits der Kausalität unterliegt und andererseits Freiheit im sittlichen Handeln besitzt, dahingehend, daß er sie dadurch radikalisierte, daß er die Natur in ihrer Selbständigkeit gegenüber dem Subjekt in dieses zurücknahm. Im Unterschied zu Kant, nach dessen Meinung die verschiedenen menschlichen Vermögen nicht aus einem einheitlichen Prinzip erklärt werden können, nahm Fichte den in der "transzendentalen Logik" zum Ausdruck gebrachten Gedanken der Identität von Erkenntnisinhalt und deren Form auf. ${ }^{9}$ Auf dieser Grundlage erweiterte er das transzendentale ICH derart, daß alle Vermögen des Menschen als Momente der Totalität des Subjekts bereits in den Ausgangspunkt ihrer Deduktion hineingenommen sind - unter der Voraussetzung der Aufhebung jeder Bindung des ICH an ein "Ding an sich". Entgegen jeglicher Analogisierung oder Identifizierung der in den Beziehungen der Menschen waltenden Logik mit den Gesetzen der Natur reflektierte und entwickelte Fichtes Philosophie in der ihr eigenen Weise die Tatsache, daß die die soziale Existenz vermittelnden Strukturen erst durch und im Handeln der Menschen konstituiert werden und dieses Handeln immer vom Wollen, Denken und Fühlen, dem Bewußtsein der Menschen bestimmt wird. Mit der intellektuellen Anschauung, dem unmittelbaren Bewußtsein, daß das Subjekt ein denkendes und zugleich handelndes Wesen ist, bestimmte Fichte das tätige Selbstbewußtsein als Voraussetzung und Bedingung allen Wissens. Im einheitlichen Erkenntnisprozeß mit seinen unterschiedlichen Stufen handelt das ICH und erkennt sich in diesem Handeln; die universell begriffene menschliche Tätigkeit ist darin als Quelle sozialkulturellen, geistigen Fortschritts benannt. 
Fichte gelang es aber nicht nur das innere Verhältnis der differenzierten Vermögen des ICH dadurch abzuleiten, daß er die Form der Erkenntnis zugleich als Form des Wollens behauptete; mehr noch: Die in der synthetischen Methode erscheinende Vernunftgesetzlichkeit oder Dialektik ist in einen alle anderen Erkenntnismethoden übergreifenden Rang erhoben. Das Prinzip des Kantschen "Naturzwecks" der Einheit von Ursache und Wir-

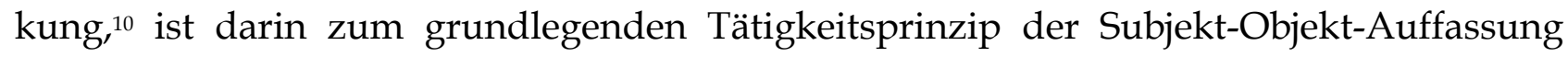
gesteigert. Der Unterschied zur mechanistischen Kausalitätsauffassung war augenfällig. Durch den Nachweis des inneren Zusammenhanges aller Momente der Erkenntnis, vermittelt über die Syntheseleistung der produktiven Einbildungskraft, gelang es Fichte, das Wissen nicht als fixe Größe, sondern als Werdendes zu fassen, worin die Momente der Erkenntnis als Stufen eines einheitlichen Prozesses auseinander ableitbar sind. Mit der auf dieser Basis konzipierten Subjekt-Objekt-Auffassung war in der Fichteschen Transzendentalphilosophie die Möglichkeit gegeben, Übergänge zwischen den Momenten der Totalität, ihr Werden in den Blick zu nehmen, war das Problem prozessierender Struktur angesprochen. Mittels der synthetischen Methode - so Fichtes Anspruch - hat die Wissenschaftslehre das Wissen logisch-genetisch darzustellen, zu entwickeln. ${ }^{11}$ Die Forderung: "Die Wissenschaftslehre soll seyn eine pragmatische Geschichte des menschlichen Geistes",12 verband die in der Dis-ussion um das Methodenmodell Descartes' erhobene Forderung nach genetischer Ableitung der Theorie mit der dem Denken eigenen geschichtlichen Dimension als eine gegensätzliche Bildungsformen durchlaufende Entwicklung.

Nun ist die durch Fichte ausgebildete Methode zur Darstellung der philosophischen Theorie als eine "limitative" oder "quantitative" Form dialektischen Denkens charakterisiert worden.13 In unter-schiedlichen Gestalten läßt sie sich ausgehend von der Platonschen Di-

\footnotetext{
10 Vgl: I. Kant: Kritik der Urteilskraft. B 286

11 Vgl.: J.G. Fichte: Ueber den Begriff der Wissenschaftslehre... In: Akad.-Aus. Bd. I/2. S. 112f., 159

12 J.G. Fichte: Grundlage der gesammten Wissenschaftslehre. In: Ak.-Aus. Bd.I/2. S. 365; ders.: Nachgelassene Schriften zu Platners "Philosophischen Aphorismen" [1794-1812]. In. Akad.-Aus. Bd.II/4. S. 46, 90; ders.: Ueber den Unterschied des Geistes, u. des Buchstabens in der Philosophie. In: Akad.-Aus. Bd. II/3. S. 334

13 Vgl.: W. Janke: Limitative Dialektik. Überlegungen im Anschluß an die Methodenreflexion in Fichtes "Grundlage" 1794/95 (GA I,2, 283-285). In: Fichte-Studien. Bd. 1. Hrsg. v. K. Hammacher, R. Schottky, W.H. Schrader. Amsterdam 1990; Autorenkollektiv: Geschichte der Dialektik. Die klassische deutsche Philosophie. Berlin 1980. S. 109; zum Gesamtproblem: K. Hammacher: Problemgeschichtliche und systematische Analyse von Fichtes Dialektik. In: Der transzendentale Gedanke. Die gegenwärtige Darstellung der Philosophie Fichtes. Hrsg. v. K. Hammacher. Hamburg 1981; J. Stahl: Fichtes Beitrag zur Ausbildung einer dialektischen Naturbetrachtung. In: Philosophie und Natur. Beiträge zur Naturphilosophie der deutschen Klassik. Collegium philosophicum Jenense. H. 5. Hrsg. v. E. Lange. Weimar 1985. S. 150
} 
airesis und der topischen Form der Logik bei Aristoteles ${ }^{14}$ durch die Geschichte der Philosophie verfolgen. Allein der Hinweis auf vorangegangene Formen besagt nichts über die bei Fichte sich findenden Eigentümlichkeiten. Diese sehe ich im Hinblick auf die Erfassung von Evolutionsprozessen insbesondere mit folgenden Momenten der synthetischen Methode bzw. transzendentalen Theorie Fichtes verbunden:

1. Der transzendentalphilosophisch begründete Gedanke der Einheit und Ganzheitlichkeit des Subjekts, seiner verschiedenen Vermögen, vor allem seines Wissens, und darüber vermittelt der Welt in ihren strukturell unterschiedlichen Bewegungsformen, geht prinzipiell auf die Aufdeckung des Zusammenhangs von Struktur und Prozeß, ihres Übergangs ineinander aus. Wissenschaftshistorisch bedeutsam ist dabei, daß Fichte unter-schiedliche Organisationstypen für die qualitativ verschiedenen Strukturformen des Universums voraussetzte, er eben nicht mehr meinte, sie reduktionistisch einer mechanischen Einheitsgesetzlichkeit unterwerfen oder - als die andere Möglichkeit - in eine qualitativ auseinanderfallende Vielheit dividieren zu müssen. ${ }^{15}$

2. Fichte vertiefte die theoretische Begründung der Freiheit des Individuums in entscheidender Weise dadurch, daß diese nicht mehr erscheint als Zufall in Gestalt der unerkannten Notwendigkeit. Stattdessen erfaßte er die Determination moralischen Handelns als (unbewußt) erzeugt durch das Handeln des Subjekts. Die Notwendigkeit steht der Freiheit damit nicht als ein Fremdes gegenüber; der Impuls für Veränderungen ist nicht gegeben als ein äußerer, sondern er ist dem Subjekt mit seinem zwecksetzenden, freien Handeln eigen. Es erzeugt selbst die es begrenzenden Bedingungen, die zugleich den Antrieb geben, sie in erneuter Tätigkeit aufzuheben und $\mathrm{zu}$ reproduzieren. ${ }^{16}$ Damit war das grundsätzliche Schema evolutionärer Bildung als sich - modern gesprochen - selbstorganisierender und

14 Vgl:: G. Schenk: Zur Geschichte der logischen Form. Berlin 1973. S. 89ff., 163ff.

15 Vgl. hierzu besonders die in Aufnahme und Auseinandersetzung mit Schellings "ursprünglichen Produktionen" der Natur in einem evolutiven Sinne ausgeformte Position der Einheit von Mensch und Natur: J.G. Fichte: Nachgelassene Schriften zu Platners "Philosophischen Aphorismen" 1794-1812. A.a.O. S. 272ff.

16 Die auch für die Philosophie der ausgehenden Aufklärung in Deutschland typischen Versuche, das Freiheitsproblem dadurch zu lösen, daß den die Menschen in ihrem Tun beherrschenden Gesetzen gegenüber denen der unbelebten Natur ein stochastischer Charakter zuerkannt wurde. hatte aber nicht vermocht, den besonderen Charakter der menschlichen Freiheit in einer einheitlichen philosophischen Ableitung, das gesamte Sein unter sich begreifend, zu leisten. Im Anspruch, dieses Problem zu lösen, besteht aber eine der wesentlichen theoretischen Intentionen Fichtes.(Vgl.: J.G. Fichte: Ueber die Würde des Menschen... In: Akad.-Aus. Bd.I/2. S. 87) 
tionärer Bildung als sich - modern gesprochen - selbstorganisierender und nicht fremdbestimmter Prozeß, des "Entstehen(s) durch ein Vergehen (ein Werden durch ein Verschwinden.)"17 begrifflich erfaßt. Entscheidend ist dabei m.E., daß das Subjekt nicht nur als eindimensionales Individuum, sondern in seinen differenten und sich widersprüchlich darstellenden Subjektbeziehungen thematisiert wurde. Das aber gehört zu den theoretischen Voraussetzungen, um - als Alternative sowohl gegenüber mechanistischen Interpretationen als auch bloß empiristischer Beschreibung - die Determinanten und Eigendynamik der jeweiligen Subjektbeziehungen in den Blick einer differenzierenden Sozial- und Subjekttheorie nehmen zu können.

3. Das Fichtesche Deduktionsverfahren geht aus von der Existenz gegensätzlicher Momente, die in der Synthese durch die produktive Einbildungskraft bewußt zur Einheit zu bringen sind. Ihre Vermittlung stellt sich wiederum als bestimmt durch neue Gegensätze dar, wodurch der Fortgang der Deduktion verbürgt ist. Die Erkenntnis der dialektischen Gegensatzbeziehung avancierte damit zum methodischen Mittelpunkt der Wesenserkenntnis. ${ }^{18}$

4. Fichte gab mit dem durch ihn unternommenen Versuch einer logischen Darstellung der Geschichte des Selbstbewußtseins einer neuen Sichtweise im historiographischen Denken insofern Raum, als er der Sache nach die Kenntnis der Genese für das logisch-theoretische Wesensverständnis einer Erscheinung für unumgänglich begründete. ${ }^{19}$ Denn die Wissenschaftslehre unterscheide sich gerade dadurch vom natürlichen Bewußtsein und werde zur Wissenschaft, daß sie die gegebene Struktur des Bewußtseins als Resultat einer Genesis auffasse, die dem empirischen Be-

17 J.G. Fichte: Grundlage der gesammten Wissenschaftslehre. A.a.O. S. 329 sowie S. 331

18 Vgl.: Wissenschaftslehre nach den Vorlesungen von Hr. Pr. Fichte. In: Akad.-Aus. Bd. IV/2. S. 107

19 Vgl.: J.G. Fichte: Grundlage der gesammten Wissenschaftslehre. A.a.O. S. 365; ders.: Ueber den Begriff der Wissenschaftslehre. A.a.O. S. 147. Für den grundlegenden Charakter dieser Einsicht spricht auch Fichtes Willen, daraus praktische Konsequenzen für die Organisation und Darstellung der Wissenschaftsdisziplinen bzw. Kunst zu ziehen. Sein Zirkular "Jahrbücher der Kunst und Wissenschaft" (vgl.: J.G. Fichte: Jahrbücher der Kunst und Wissenschaft [1800]. In: Akad.-Aus. Bd. I/6. S. 425f.) weitete den Gedanken, daß die theoretische Philosophie eine pragmatische Geschichte des menschlichen Geistes sei, auf die Gesamtheit der theoretischen und künstlerischen Aneingungs- und Ausdrucksweisen des Menschen aus. (Vgl.: J. Stahl: Ästhetik und Kunst in der Transzendentalphilosophie Johann Gottlieb Fichtes. In: Philosophie und Kunst. Kultur und Ästhetik im Denken der deutschen Klassik. Collegium philosophicum Jenense. H. 7. Hrsg. v. E. Lange. Weimar 1987. S. 83) 
wußtsein verborgen bleibe. ${ }^{20}$ Auf einige damit verbundene bedeutsame Aspekte im Hinblick auf die weitere Ausbildung entwicklungstheoretischen Denkens sei in diesem Zusammenhang, da sie in der Literatur umfangreich behandelt sind, nur andeutungsweise eingegangen: Zum einen, daß, da das menschliche Bewußtsein immer in der Einheit seiner Momente fungiert, folglich auch immer das ICH als eine sich differenzierende Einheit von Subjekt und Objekt den Ausgangspunkt der theoretisch-logischen Ableitung bilden muß. Die Konsequenz bezüglich der Theoriedarstellung lautet: Ausgang von einem Anfang, aus dem sich alle anderen Momente der Totalität deduzieren, entwickeln lassen.. ${ }^{21}$ Zum anderen, sich aus dem vorigen Gesichtspunkt ableitend, die Auffassung, daß die Produktion (philosophischen) Wissens im Verlaufe der Geschichte nicht zu völlig unvermittelt zueinander stehenden Resultaten führt, sondern diese wesentliche Stufen ihrer Bildung darstellen. Demzufolge hat die Geschichte des Selbstbewußtseins zugleich den historischen Wechsel der philosophischen Systeme in sein inneres Wesen aufzulösen.22

5. Schließlich sei noch generalisierend auf Fichtes transzendentale Reflexion der Rolle des menschlichen Subjekts im Erkenntnisprozeß hingewiesen. Die von ihm thematisierten reflexiven Begründungsstrukturen menschlichen Erkennens führten Fichte nicht nur zur Auffindung der zyklischen Struktur des Wissens, der Betonung seiner Relativität und prinzipiell un-endlichen Erweiterbarkeit im Gegensatz zum harmonisierenden Vollständigkeitsideal der Ordnungswissenschaften. Die in der transzendentalphilosophischen Begründung der Erkenntnis ausgesprochene Problematik offenbarte sich in ihrer Relevanz sowohl in der im 19. Jahrhundert einsetzenden ideologiekritischen Analyse als auch der Diskussion um die Stellung des Subjekts im Prozeß natur- und sozialwissenschaftlicher Erkenntnis im Zusam-

\footnotetext{
${ }^{20}$ Vgl.: J.G. Fichte: Grundlage der gesammten Wissenschaftslehre. A.a.O. S. 364f.; weiterhin die "GRADATION der Fortschreitung unserer Vernunft und Entwicklung unsers Bewußtseyns" in: Wissenschaftslehre nach den Vorlesungen von Hr. Pr. Fichte. A.a.O. S. 26

${ }^{21}$ Vgl. u.a.: J.G. Fichte: Ueber den Begriff der Wissenschaftslehre... A.a.O. S. 125, 130ff.; ders.: Versuch einer neuen Darstellung der Wissenschaftslehre. A.a.O. S. 205

22 Vgl.: Ueber den Begriff der Wissenschaftslehre... A.a.O. S. 143; dazu: J. Stahl: Philosophiehistorische Auffassungen in der theoretischen Philosophie Johann Gottlieb Fichtes. In: Deutsche Zeitschrift für Philosophie. 34.Jg. (1986) H.6. S. 532f.
} 
menhang mit der Ausarbeitung der Quantenmechanik oder moderner Kognitionstheorien. ${ }^{23}$

Fraglos stellt Fichtes Philosophie ein herausragendes Zeugnis des auf philosophischer Ebene einsetzenden Aufbrechens mechanizistischen Denkens dar. Aber deswegen sollte nicht Fichtes notwendiges und keineswegs negativ zu begreifendes Verhaftetsein im Denken seiner Zeit übersehen werden, ließ die Wissenschaftslehre nicht zukünftige Denkanstrengungen zum theoretischen Begreifen der Evolution in Natur, Gesellschaft und im Denken gegenstandslos werden. So unterliegt das in den Axiomen der klassischen Mechanik sich ausdrückende Verständnis der Wirkungsweise von Gesetzen nicht nur weitgehend der Fichteschen Philosophie; bis weit in das 20. Jahr-hundert prägt es das rationalistischen Auffassungen verpflichtete Weltbild. Die sich bei Fichte genauso wie bei Schiller, Herder, Schelling oder Hegel findende weitgehend bildhafte bzw. empirische Beschreibung der widerstreitenden Tendenzen sozialer Gärung sind sicherlich vorrangig in ihrer Leistungsfähigkeit zur Erfassung der Kompliziertheit des sozialen Geschehens gegenüber linearen Fortschrittsauffassungen zu werten. Andererseits bieten sie eben nur bedingt ein theoretisch-begriffliches Modell des Abbaus und der Neubildung sozialer Strukturen in Umbruchphasen. Exemplarisch sei an dieser Stelle folgende Einsicht Fichtes zitiert: "Jene Errichtung einer rechtlichen Verfassung im Innern aber, und die Befreiung des ersten Volks, das da wahrhaftig frei wird, erfolgt nothwendig aus dem stets wachsenden Drucke der herrschenden Stände auf die beherrschten, so lange, bis er unleidlich wird; - ein Fortschritt, welchen man den Leidenschaften und der Verblendung jener Stände, auch wenn sie gewarnt werden, sehr ruhig überlassen kann." ${ }^{24}$ Sie bringt wie das Bild des Theaters von Schelling oder Hegels "List der Vernunft" vor allem die Gewißheit von der Durchsetzungsfähigkeit der bürgerlichen Gesellschaft zum Ausdruck. Allen gemeinsam ist die Überzeugung von der Unabdingbarkeit des historischen Fortschritts - ganz dem klassischen Kausalverständnis verpflichtet.

\footnotetext{
${ }^{23}$ Vgl.: H. Maturana: Erkennen, die Organisation und Verkörperung von Wirklichkeit. Ausgewählte Arbeiten zur biologischen Epistemologie. Braunschweig/Wiesbaden 1982; H. Maturana/F. Varela: Der Baum der Erkenntnis. Die biologischen Wurzeln des menschlichen Erkennens. Bern/München/Wien 1987.

24 J.G. Fichte: Die Bestimmung des Menschen. In: Akad.-Aus. Bd. I/6. S. 274, ebenfalls S. 273 sowie ders.: [Rezension:] Zum ewigen Frieden... In: Akad.-Aus. Bd. I/3. S. 221, 227
} 
Vor dem Hintergrund des Spannungsverhältnisses von in der Wissenschaftslehre formulierten Ansprüchen an das Handeln der Subjekte und der Geschichtsrealisation als offenem Prozeß sozialer Evolution entfaltete Fichte seine Perspektivkonstruktionen des Lebens in der bürgerlichen Gesellschaft. Deren prinzipielle humanistische Intentionen sind unbezweifelt. Doch indem darin die Beschränkung des Individuum auf sein Dasein als Citoyen zum systemtragenden Vorzug des auf egalitaristischen etatistischen Prinzipien beruhenden Sozialgebildes erhoben ist, geht Fichtes Vorstellung von der bürgerlichen Gesellschaft auf ein harmonisierendes, deren innere Triebkräfte und Eigendynamik verfehlendes und letztlich statisches Gesellschaftsmodell aus. ${ }^{25}$

Nicht in diesen sozialen Alternativvorstellungen liegt m.E. die aktuelle Dimension der Fichteschen Transzendentalphilosophie begründet. Im Hinblick auf die theoretische Erfassung von Evolutionsprozessen sehe ich diese insbesondere verbunden mit Fichtes Akzentuierung des Subjekts, seines vernunftbestimmten Handelns in der Entfaltung, Realisation seiner Individualität als der evolutiven Konstituante sozialer Strukturbildung. Des weiteren sehe ich eine historisch keineswegs schon abgegoltene Leistung der Fichteschen Transzendentalphilosophie in deren Aufdeckung der Spezifik menschlicher Subjektivität, resultierend aus der kognitiven wie sozialen Tätigkeit des Menschen. Sie richtet sich gegen jede Beschneidung der dem Menschen eigenen theoretischen und praktischen Fähigkeiten wie sie sich verbindet mit jeglicher Form von mechanizistischem Reduktionismus und Objektivismus oder auch der scheinbaren "Sachzwanglogik" der Entwicklung technisch-ökonomischer, geistig-kultureller oder sozialpolitischer Systeme. Der Hinweis auf den Mythos einer von der Hyperintelligenz künstlicher Wissenssysteme beherrschten Zukunftsgesellschaft mag hier genügen.

${ }^{25}$ Vgl.: J.G. Fichte: Ueber StaatsWirthschaft. In: Akad.-Aus. Bd. II/6. S. 7 